\title{
Síndrome Isquêmica Aguda Cerebral e Miocárdica Concomitante Associada a Infecção por SARS- CoV-2: Um Relato de Caso
}

\author{
Milana Gomes Prado ${ }^{1 *}$, Amanda Silva Fraga ${ }^{1}$, Ricardo Peixoto Oliveira ${ }^{1}$, Joberto \\ Pinheiro Sena ${ }^{1}$, Bruno Macedo de Aguiar ${ }^{1}$, José Carlos Raimundo Brito ${ }^{1}$ \\ Serviço de Cardiologia do Hospital Santa Izabel; Salvador, Bahia, Brasil
}

Correspondence addresses: Dra. Milana Gomes Prado milanagprado@gmail.com

Received: April 3, 2020

Revised: May 5, 2020

Accepted: May 9, 2020

Published: May 31, 2020

Data Availability Statement: All relevant data are within the paper and its Supporting Information files.

Funding: This work was the result of authors' initiative. There was no support of research or publication funds.

Competing interests: The authors have declared that no competing interests exist.

Copyright

(C) 2020 by Santa Casa de Misericórdia da Bahia. All rights reserved.

ISSN: 2526-5563
Logo após declarada a pandemia pelo SARS-CoV-2 em março de 2020 pela Organização Mundial de Saúde (OMS), acreditava-se ser um vírus que acometia unicamente os pulmões podendo levar à pneumonia e à Síndrome Respiratória Aguda Grave. Entretanto, com o passar do tempo, novas descobertas foram feitas e viu-se tratar de uma doença sistêmica que predominantemente caracteriza-se por altos graus de processos inflamatórios e trombóticos. Neste relato, descrevemos um paciente com acidente vascular cerebral (AVC) isquêmico concomitante a infarto agudo do miocárdio (IAM) silencioso e miopericardite.

Palavras-chave: SARS-CoV-2; Inflamação; Trombose; AVC; IAM.

Shortly after the SARS-CoV-2 pandemic was declared in March 2020 by the World Health Organization (WHO), the scientists believed that the virus affected only the lungs and could lead to pneumonia and Severe Acute Respiratory Syndrome. However, over time, the discoveries suggested being a systemic disease, which is predominantly characterized by high degrees of inflammatory and thrombotic processes. In this report, we describe a patient with ischemic stroke concomitant with silent acute myocardial infarction (AMI) and myopericarditis.

Keywords: SARS-CoV-2; Inflammatory; Thrombosis; Stroke; AMI.

\section{Instrodução}

No final de 2019, o mundo presenciou o surgimento de uma nova condição infecciosa de rápida disseminação, a infecção por SARSCoV-2. Inúmeros relatos vêm sendo publicados relacionando a infecção pelo novo coronavírus com apresentações clínicas diversas, além da doença respiratória per si. Patologias reconhecidamente prevalentes como acidente vascular encefálico e infarto agudo do miocárdio podem estar relacionadas a processos inflamatórios e trombóticos desencadeados pelo vírus. ${ }^{1}$ A lista de manifestações clínicas e condições relacionadas continuam sob processo de aprendizado, em decorrência do reconhecimento recente do vírus e alguns de seus mecanismos de ação. ${ }^{2}$ Este relato de caso traz o caso de um paciente com infecção por SARS-CoV-2 que apresentou acidente vascular encefálico isquêmico concomitante a infarto agudo do miocárdio silencioso e miopericardite. 


\section{Relato de Caso}

Paciente do sexo masculino, 51 anos, hipertenso, diabético, obeso, foi admitido em 27/05/2020 em unidade de emergência com relato de desvio de comissura labial à direita e hemiparesia esquerda, com duração superior a 12 horas, sugestivo de Acidente Vascular Encefálico Isquêmico fora de janela de trombólise, sendo encaminhado para hospital terciário. Referiu quadro de febre não aferida, tosse e mialgia iniciada há uma semana, com melhora dos sintomas à admissão. Realizado teste rápido para COVID-19 (IgM/IgG), com resultado positivo para doença aguda. Foi admitido em nosso setor com dados vitais dentro dos limites da normalidade, exceto por taquicardia FC 120bpm. Bom estado geral, afebril, normocorado, vigil, pupilas isocóricas e fotorreagentes, disartria leve, desvio de comissura labial à direita, hemiplegia e hemianestesia à esquerda. Sem outros achados importantes no exame físico segmentar.

Paciente foi submetido à tomografia de crânio (Figura 1) em 28/05 que demonstrava área de hipodensidade em topografia temporoparietal

Figura 1. Tomografia de crânio apresentando hipodensidade em topografia temporoparietal e insular direita.

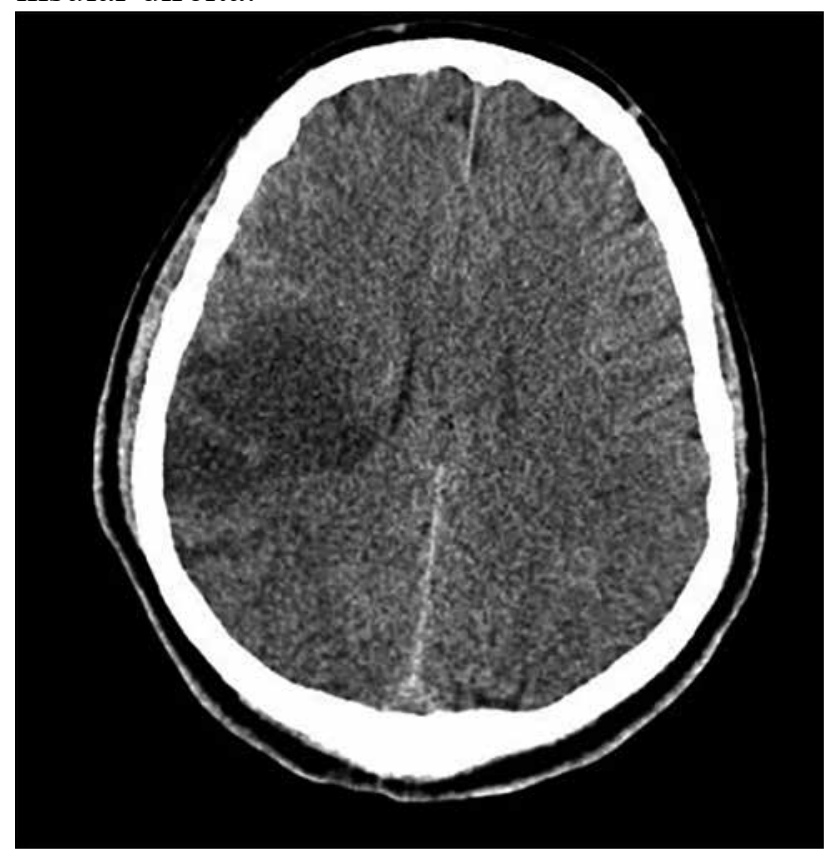

e insular direita, inferindo evento isquêmico subagudo. Optou-se por tratamento clínico conservador da isquemia cerebral.

Realizou exames complementares, dentre eles, eletrocardiograma, que evidenciou supradesnivelamento de segmento ST difuso (Figura 2) e troponina co valor de $6,030 \mathrm{ug} / \mathrm{mL}$, seguido de 2,0 ug/mL (VR: 0,160ug/L).

Foi realizada radiografia de tórax (Figura 3), sem alterações. Mantinha-se sem suplementação de $\mathrm{O}_{2}$.

Submetido à tomografia de tórax em 30/05 (Figura 4), com infiltrado pulmonar intersticial bilateral.

Optou-se por realizar coronariografia (Figura 5) que demonstrou: descendente anterior com estenose segmentar de $50 \%$ no segmento proximal (placa de aspecto angiográfico instável), oclusão no segmento distal, com aspecto de oclusão recente por trombo (macroembolização distal). Demais segmentos com discretas irregularidades parietais. Circunflexa apresenta discretas irregularidades parietais. Coronária

Figura 2. Eletrocardiograma admissional em ritmo sinusal; FC 148 batimentos; supra desnível do ST difuso.

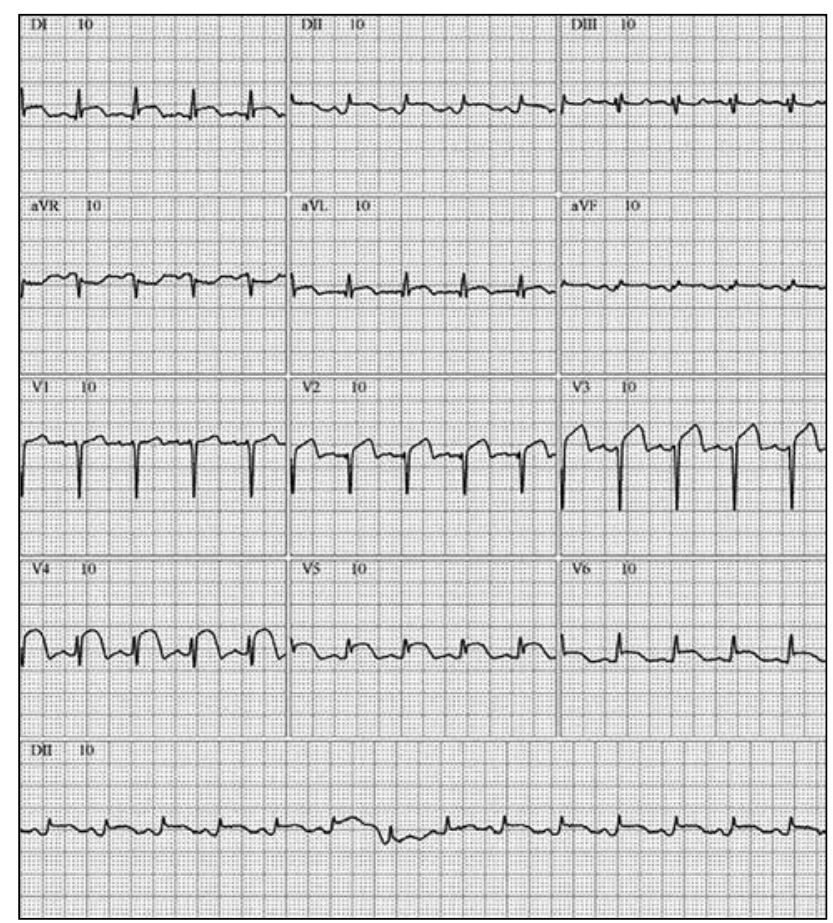


Figura 3. Radiografia de tórax (AP) realizada com dificuldade técnica. Pulmões com transparência satisfatória, ângulos basais laterais livres, com discreto aumento da relação cardiotorácica.

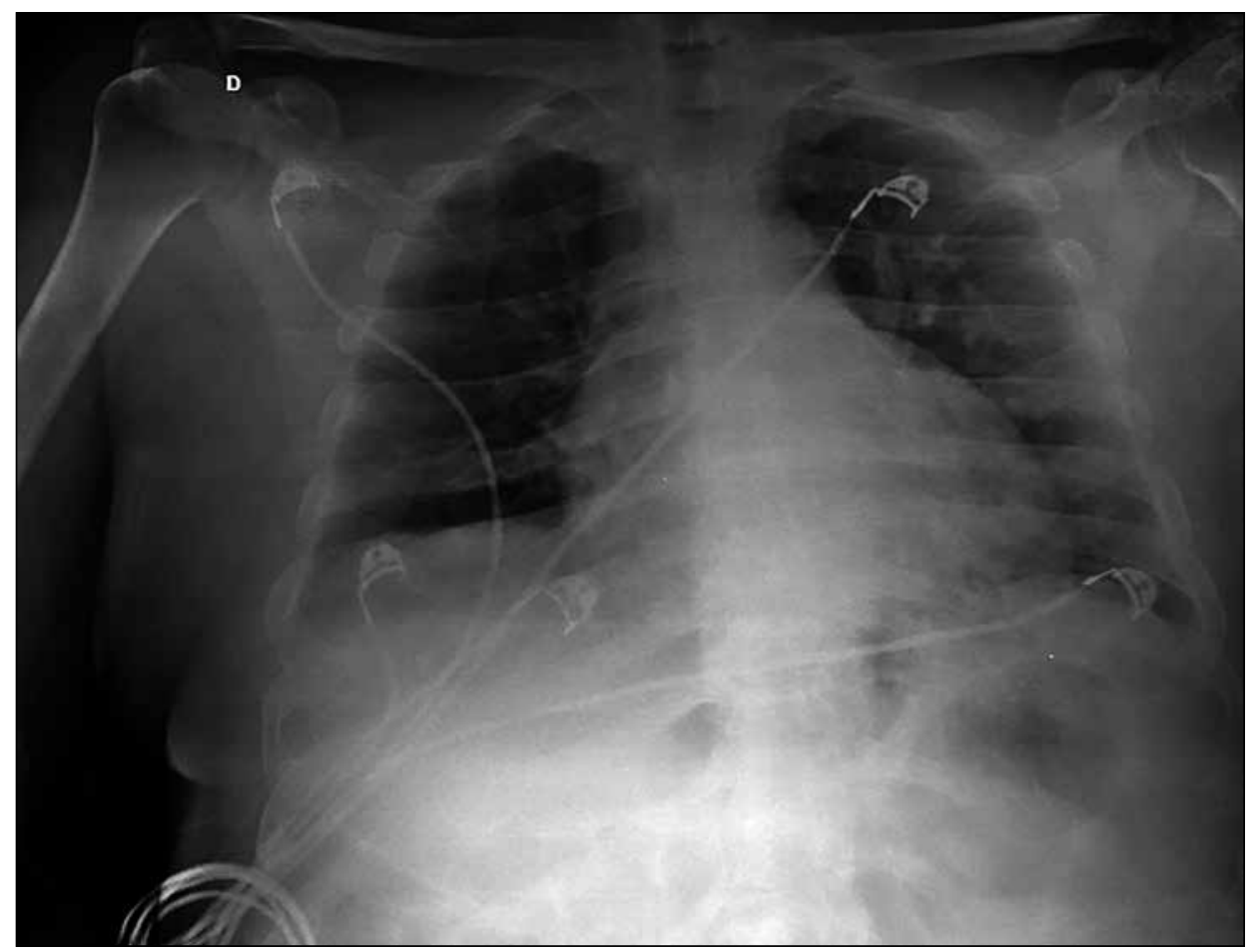

Figura 4. Tomografia de tórax sem contraste. Opacidades multifocais, com atenuação em vidro fosco em ambos os pulmões, predominando nas regiões periféricas e inferiores, notando-se, ainda, algumas opacidades peribroncovasculares e periféricas, por vezes configurando pneumonia oliterante em organização (BOOP), envolvendo entre $25 \%$ e $50 \%$ do parênquima pulmonar.

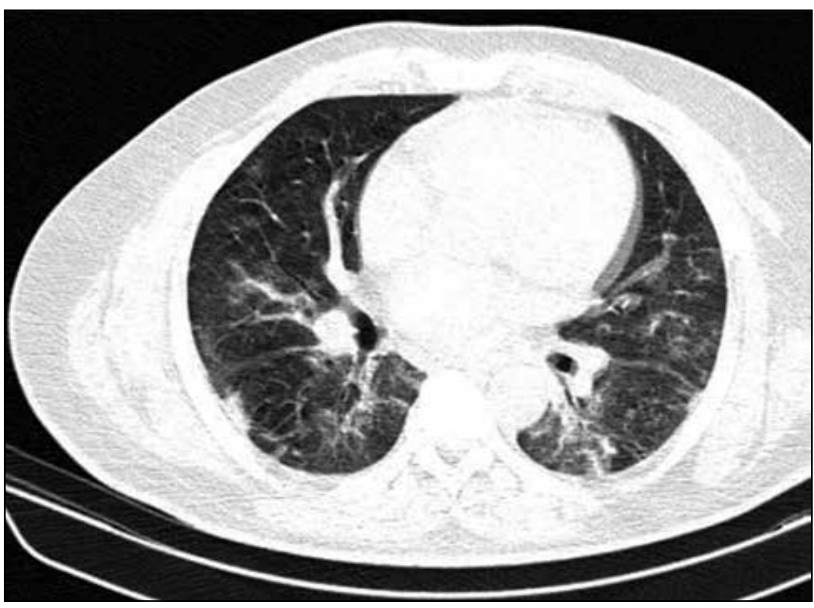

direita dominante, com estenose de $25-50 \%$ no segmento proximal e ateromatose leve nos demais segmentos. A ventriculografia mostrava ventrículo esquerdo com volume diastólico final aumentado $++/ \mathrm{IV}$, apresentando discinesia

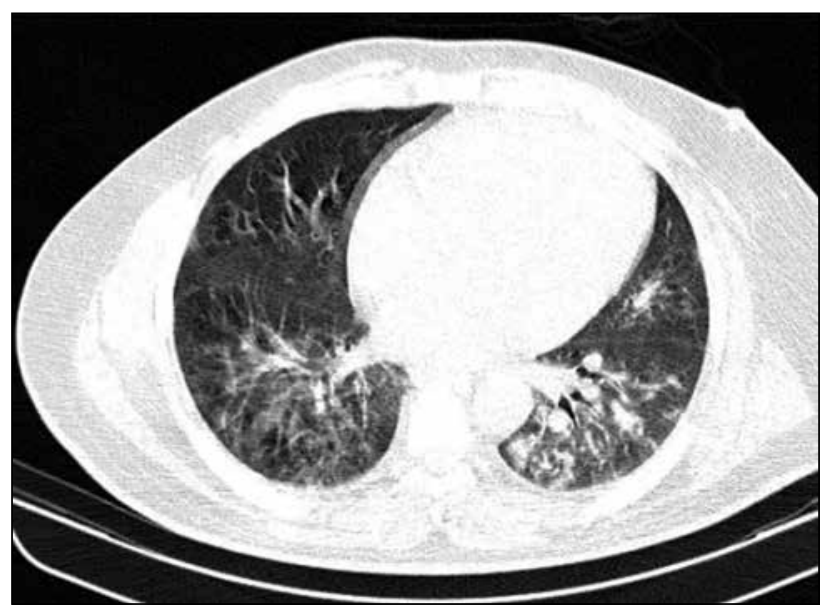

ântero-apical e apical com hipocinesia moderada ântero-medial.

Iniciada dupla antiagregação plaquetária (DAPT). Nova tomografia de crânio de controle evidenciou pequena transformação hemorrágica 
Figura 5. Cateterismo cardíaco.
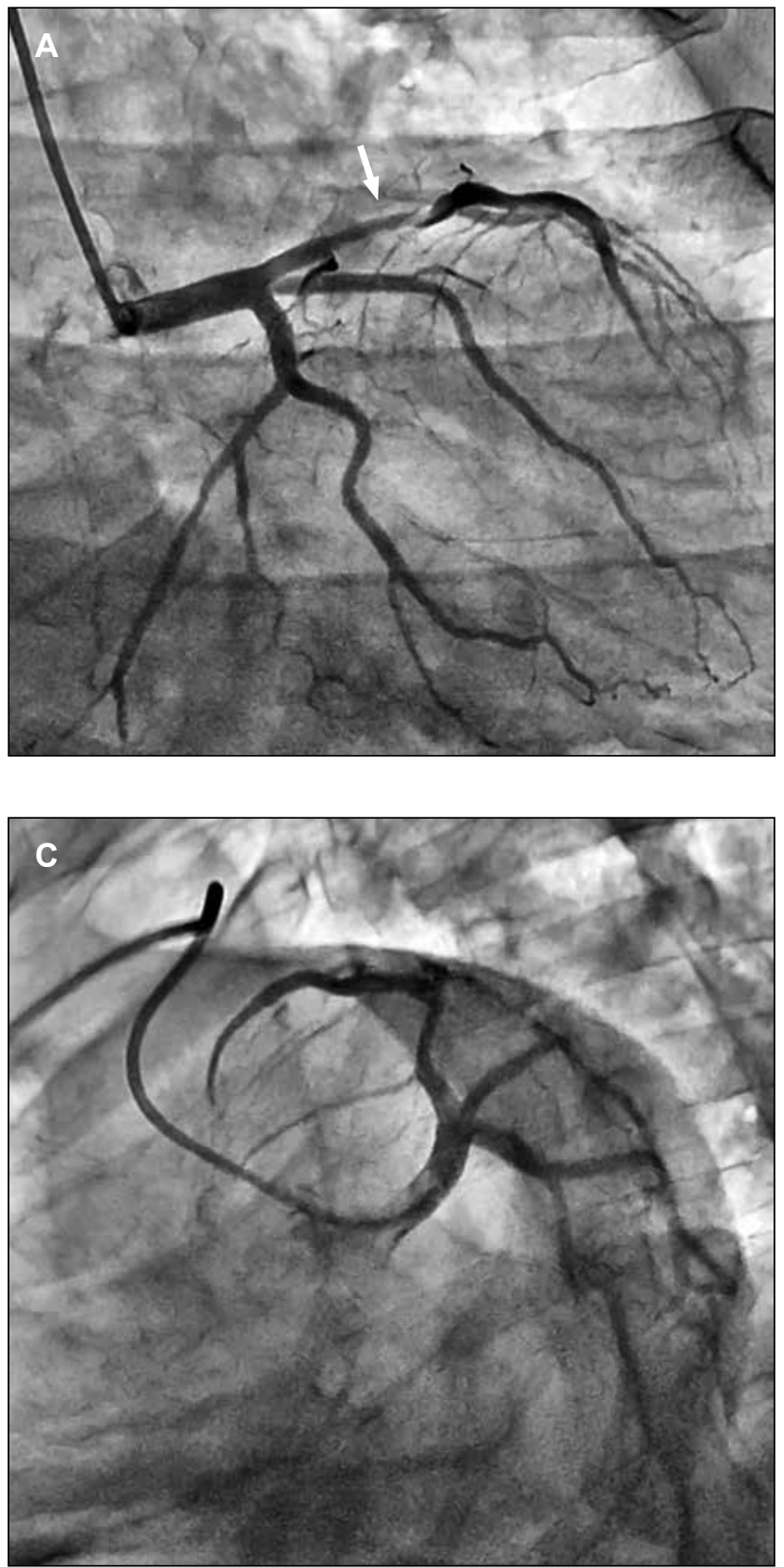

petequial, optando-se por aguardar janela de segurança para introdução de anticoagulante, sendo suspenso DAPT.

Realizou posteriormente ecocardiograma transtorácico (30/05), que mostrou: ventrículo esquerdo com função sistólica diminuída (FEVE 35\%), secundária à acinesia apical de todas as paredes e hipocinesia em demais paredes. Movimentação atípica do septo ventricular. Não foi possível análise da função
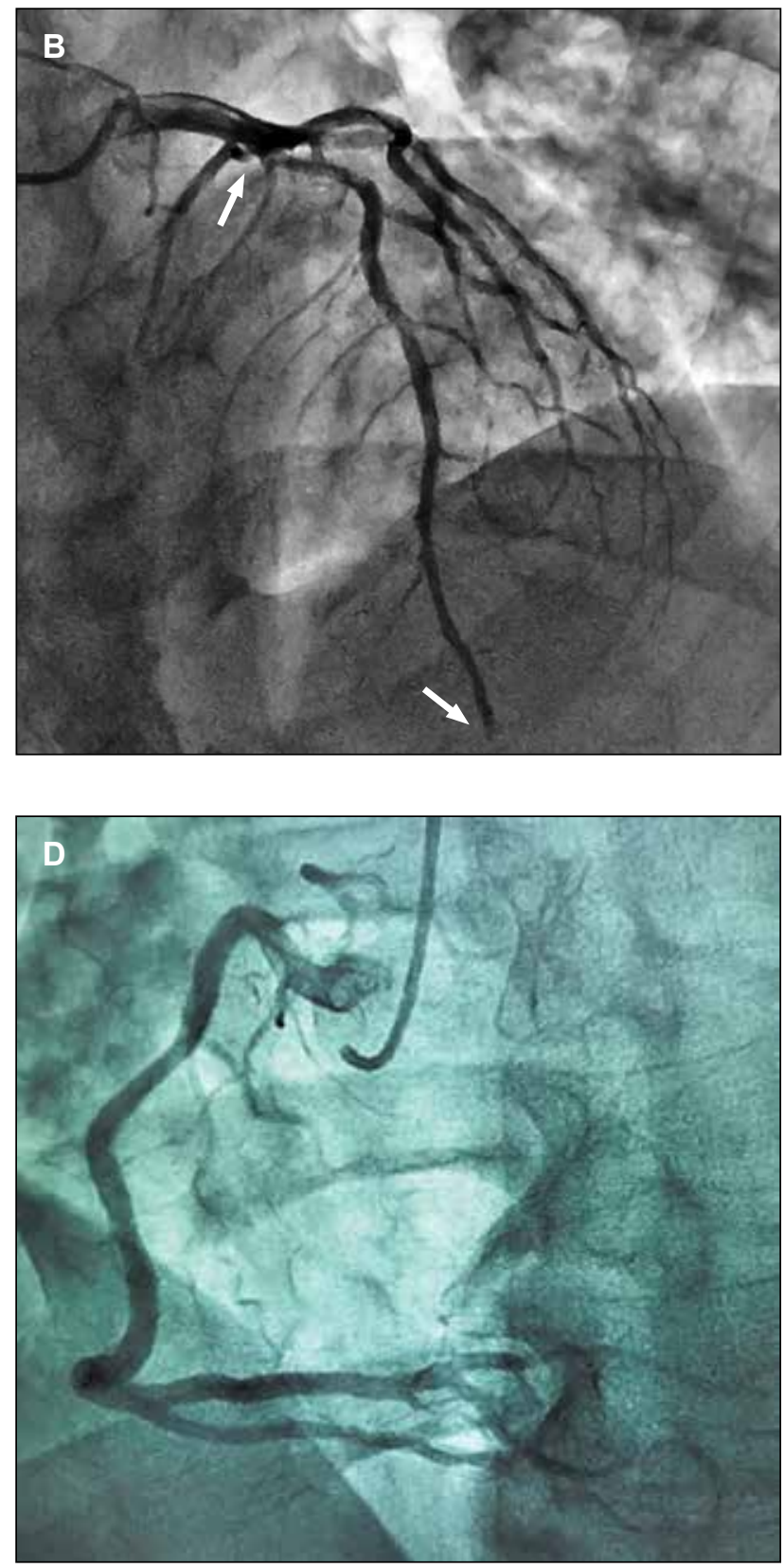

diastólica $(\mathrm{FC}=120 \mathrm{bpm})$. Ventrículo direito apresentava função sistólica normal.

Paciente permaneceu hemodinamicamente estável, eupneico em ar ambiente, com déficit em dimídio esquerdo. Recebeu alta após 12 dias de internamento hospitalar em uso de Clopidogrel $75 \mathrm{mg} /$ dia, Rivaroxabana 20 $\mathrm{mg} / \mathrm{dia}$, Metoprolol 100mg/dia, Losartana $100 \mathrm{mg} / \mathrm{dia}$, Atorvastatina $80 \mathrm{mg} / \mathrm{dia}$, Insulina NPH 12 UI/dia. 


\section{Discussão}

A injúria miocárdica tem sido tema de investigação dentro do espectro de manifestações da doença causada pelo SARS-CoV-2.,4 Esta complicação tem sua prevalência variando entre $7,2 \%$ a $12 \%$ dos pacients. O mecanismo sugerido é de lesão miocárdica por invasão direta do vírus nos cardiomiócitos ou por elevação de marcadores inflamatórios.

Estudo anterior buscou correlacionar as características e estado clínico dos pacientes com COVID-19 e injúria miocárdica associada. $\mathrm{O}$ perfil descrito foi de pacientes com idade avançada, múltiplas comorbidades, níveis elevados de marcadores de necrose miocárdica e resposta inflamatória. Além disso, percebeu-se que o acometimento miocárdico não é incomum, assim como a lesão miocárdica, principalmente entre os que falecem. ${ }^{5}$

$\mathrm{O}$ caso descrito suscitou dúvidas quanto à presença de uma síndrome coronária aguda (IAM com supra de ST) associada ao quadro de AVC e COVID-19, pois não havia relato de sintomas relacionados à isquemia miocárdica. No entanto, $\mathrm{o}$ paciente reunia diversos fatores de risco para doença arterial coronariana, alterações eletrocardiográficas que poderiam sugerir, além da suspeita diagnóstica de miocardite, IAM de parede anterior e elevação expressiva de troponina. Pela possibilidade de mudança de conduta no que tange ao tratamento apropriado ao quadro em questão (SCA), visando a redução de desfechos clínicos significativos, optou-se, após ampla discussão médica avaliando o risco-benefício do procedimento, pela realização de uma coronariografia. Prevaleceu, portanto, como principal hipótese da injúria miocárdica sofrida pelo paciente, o diagnóstico de IAM tipo 1, após confirmação no CATE de placa ateromatosa obstrutiva no segmento proximal da artéria descendente anterior, com critérios angiográficos de instabilidade, associada à oclusão coronariana por provável macroembolização distal de trombo e demonstração ecocardiográfica de alteração segmentar da região correspondente (paredes ântero-médio apical e apical). Entretanto, a alteração eletrocardiográfica de lesão subepicárdica que avançava além da delimitação das paredes acometidas pelo IAM (supra de ST difuso), com lesão inflamatória em átrios (infra de PR), levantou a suspeita de miopericardite concomitante, como causa da injúria miocárdica, nesse paciente com COVID-19. ${ }^{6}$

A hipocinesia do ventrículo esquerdo, sem correspondência anatômica com a lesão oclusiva culpada, associada à possível pericardite e elevação de marcador de necrose miocárdica, corrobora para a suspeita de miopericardite. Faz-se necessário destacar que o paciente em questão não apresentou sintomatologia típica de acometimento cardíaco ou seus equivalentes, fato que pode ser prevalente em pacientes diabéticos reportando como fator subjacente a diferença no processamento neural central e periférico da dor. O diagnóstico de IAM com supra anterior silencioso recente poderia justificar o quadro clínico de AVCi, observado na admissão do paciente, pelo mecanismo conhecido de embolização sistêmica de trombo intracavitário, formado no ventrículo esquerdo após o infarto.

\section{Referências}

1. Arthur Rente A, Uezato D, Uezato KMK. Coronavirus and the Heart $\mid$ A Case Report on the Evolution of COVID-19 Associated with Cardiological Evolution. Arq Bras Cardiol. 2020;114(5):839-842.

2. Mao L, et al. Neurological Manifestations of Hospitalized Patients with COVID-19 in Wuhan, China: a retrospective case series study. MedRxiv 2020.02.

3. $\mathrm{Xu} \mathrm{XW}, \mathrm{Wu} \mathrm{XX}$, Jiang $\mathrm{XG}$, et al. Clinical findings in a group of patients infected with the 2019 novel coronavirus (SARS-Cov-2) outside of Wuhan, China: retrospective case series BMJ. 2020; Feb 2019.

4. Neto, et al. Coronavirus Disease 2019 and the Myocardium, Arq Bras Cardiol. 2020; 114(6):10511057.

5. Shi S, et al. Characteristics and clinical significance of myocardial injury in patients with severe coronavirus disease 2019. European Heart Journal. 2020.

6. Montera MW, Mesquita ET, Colafranceschi AS, Oliveira Junior AM, Rabischoffsky A, Ianni BM, et al. Sociedade Brasileira de Cardiologia. I Diretriz Brasileira de Miocardites e Pericardites. Arq Bras Cardiol 2013;100(4 supl. 1):1-36. 